\title{
Teachers' perceptions and efficacy to deal with adolescent students' behaviour disorder in schools of low-income area of Karachi
}

\author{
Aziz-un-Nisa* \\ Naila Siddiqua** \\ Syed Moinuddin Peerzada***
}

\begin{abstract}
This qualitative study is to investigate the teachers' perception and efficacy to deal with adolescent students' behaviour disorder in schools of low-income areas of Karachi. It is observe that when children enter the age of 12 or 13 some emotional, hormonal and physical changes occur in them. Which in turn results in the behavioural changes in them. This study aim to investigate that whether the teachers of these age group students in low-income areas of Karachi have proper awareness of the causes and reasons of the behavioural disorders of these students and up to what extent they are capable of dealing with such students. For the purpose of study, interviews of three teacher from three different schools situated in various parts of Karachi were conducted. The study revealed that the teachers of low-income areas of Karachi are well aware of the behaviour problems of adolescent students and they are quite capable of dealing students with major behaviour problems. It is evident that physical and psychological changes occur with the start of the adolescent. Due to these changes students' thought process are changed. They want their self-respect; boys become more confident while girls become diffident and reserved. If not dealt properly they may become aggressive, disobeying, lack of seriousness in their studies etc. These behaviour problems can disturb the classroom environment. Proper counseling, motivation, negative reinforcement, appreciation, proper care, appropriate punishments if necessary, involving them in-group activities, making them responsible and adopting friendly behaviour are some strategies suggested by the respondents to deal with such students.
\end{abstract}

Key words: adolescent, behaviour disorder, teachers' efficacy, teachers' perceptions

*Assistant professor, Department of education, uok

**Assistant professor, Department of education, uok

*** Ph.d research scholar 


\subsection{Introduction:}

With the start of the adolescence some emotional and behavioral problems are observe in the students. According to Butler, some of these are aggression, disobedience, rule breaking, anxiety, depression, social skills deficits, social withdrawal, and attention deficits. Management of students' behaviour is one of the present day issues being faced by the school community. Behaviour problems such as bullying, violent behaviour in school and other such bad behaviour issues result in a poor atmosphere for the school community and a sense of fear and frustration in the school. Many researchers have argued that in these situations, school can become unsafe.

Troublesome student behaviour can affect teachers and the students in the school environment. These students may disrupt lessons or even make it impossible to continue, while on the other hand, teachers may feel "burned out" or compelled to leave the profession. That is why, one indicator of successful teachers in middle and high school is the quality of their behaviour management skills.

\subsection{Review of Related Literature:}

Adolescents are people with specific psychological health concerns and needs. This is the age of beginning of serious mental problems like depression and psychosis. Over load of stress from physical, emotional, sexual and social changes make these children overloaded with stress resulting in aggression, anxiety, poor social skills and poor physical health.

The adolescents may display behaviour such as complaining, refusal to obey rules and ignoring instructions, lying, physical or oral abuse, destructiveness and even criminal activities. A community study conducted in Lahore to investigate the prevalence of emotional and behavioral problems in schoolchildren. This study revealed a prevalence of $9.3 \%$ with anti- social problems being the most common. A recent study to find the referral pattern of child with EBD to a child psychiatric clinic at a university hospital of Karachi reported that the most common reason for referral is aggressive behaviour.

The behavioral issues result in the wastage of time for all the students in the class, disturbance in the learning environment. This indiscipline also leads to poor academic achievements of the students with behavioural issues and its ripple effect also affects the learning of normal students. Inappropriate behaviour in schools can affect both students and teachers. If teachers are not confident to manage such behaviour, they mostly apply strategies with no conceptual consideration. Besides this, they implement strategies ineffectively which results in the continuation of students' problem behaviour or even the increase in the behaviour problem. 
The behaviour problem is major problem among students of secondary schools in Nigeria. Troublesome behaviour is an issue of concern to schools, parents and to the peers, whose education may be affected badly. Therefore, disturbing behaviour cannot be overlooked, and schools must adapt an implicit and sound behaviour and discipline policy. In secondary schools, the situation of behaviour problem is poorer because the students as adolescent become aware of their rights to privacy, belief, opinion, and expression etc. Serious breaches of school discipline policy, assault on teachers and peers, use of abusive and offensive language, harassment, threats and bullying of peers and teachers, use of drugs and intrusion into the school or classrooms to confront the teacher are observed in different situations by these students.

In any school day, a student's struggle with his/her behaviour and emotions may affect the prepared academic lessons. The researcher has faced this personally as he had taught adolescent students with behavioural difficulties. This lack of attention to academics by troublesome students can result in less academic time in the classroom. This waste of time can make the teacher to feel anxiety, insecurity, and distrust in his/her teaching abilities. Research over the past three decades has established that a teacher's efficacy is related to various factors associated to academic achievement. Teacher efficacy (TE), a teacher's confidence in his/her ability to influence student learning, is connect to both student and teacher characteristics and outcomes.

Students being a social entity are in a position where there role models should not be lacking things like, social elegances and good communication skills. Most of the students take their teachers as role models for refining the ability of building relationships. Likewise they use the school as a practice ground for the development of their social skills. In addition, in schools, teachers play a dominant role in developing appropriate behaviour for social skills, which are necessary to nurture a society of young people who are responsible and law-abiding citizens.

Researches about the reasons of recurrence of behaviour problems in adolescence state that this is a stressful period of life, because it relates to various worrying factors like, academic, social and environmental. All these factors generate anxiety and strain for them. And most of the adolescents are not able to release these tensions by expressing their state of mind. Accordingly, this tension is reflected in their behaviour at home and school. This disturbing behaviour are problematic for parents and the teachers as well. In addition, the classroom environment suffered due to this behaviour disorder. Proper mediation is therefore vital in the classroom to overcome disturbing behaviour in classroom and eliminate the associated effects on teachers and students.

The professional development and preparation of teachers and school environment 
are the most effective bases for the beliefs of teachers to handle misbehavior of students. Many teachers make personal efforts to learn the skills to manage students' behaviour disorders as their prior knowledge is limited. Besides this, teachers also emphasize the need of support from the principal and colleagues to develop their understanding about the management of these behaviour issues.

Conchas \& Vigil amined the culture of the schools and the issues which prevent adolescent boys from social mobility and reported that the boys from Black, Latino or Asian origin are deprived in the modern school system due to their skin colour. Due to this, they skip out of school and join in gang activities or start using drugs or alcohol.

Adolescent boys commonly exhibit psychological indications when one of their parents die. They keep their feelings within themselves. Whereas girls experience low self-esteem and become reserved and shy. After demise of parent another family member are unable to help adolescent in handling this sorrow effectively. In this context, honest adults become important and teachers can play a vital role to heal this state of grief and sorrow.

\subsection{Statement of the Problem:}

Severe violations of school discipline policy including attack by students on peers and teachers, verbal abuse, disgusting language against teachers and fellow students, sexual and other forms of harassment, threat and pressure on students and teachers, keeping weapons, supplying / using drugs, and interventions into the school or classrooms by adults to threat the teachers or administration. This dilemma has now become alarming and endangering the administration of the secondary schools. The management of such disciplinary problems in schools needs earnest consideration.

The population of adolescent in Pakistan is to be about 27 million in 2000, and anticipated to increase, up to 44.6 million by the year 2020. This is an increase of $39 \%$ in just 20 years. This age group is more or less one fourth of the population in Pakistan. Adolescents are a distinctive population with certain health matters and needs. This is the peak age of start for serious emotional ailments like stress and psychosis. Over burden of stress from sexual, emotional, social, and physical changes makes teenagers overloaded with tension resulting in anxiety, alienation, anger, poor public dealing abilities and actual physical illness. This period is described by its physical and psychological changes in the youngster, with increasing demands from and influence of peers, school and wider society. It is well known that behaviour developed during this period effect health in adulthood. Various health endangering behaviour (e.g. smoking, alcohol, drugs) besides health augmenting behaviour (e.g. physical exercise) assumed in adolescence and they often continue into adulthood. 
The secondary schools in Karachi are also facing disciplinary and misbehavior problems from the adolescent students specially, in grades viii, ix and $\mathrm{x}$. This problem not only affects the learning outcomes of these students but also the school, teachers, parents and even the society are affected badly by the misbehavior of these students if not dealt appropriately. The efficacy of teachers to deal with such students plays a vital role in maintaining the discipline, changing the emotional and behaviour manners of the students. It help to develop positive attitudes in these students. This study is aimed to learn about the efficacy and perception of teachers and strategies to improve their efficacy to deal with students with emotional and behaviour disorders. The study conducted in the three private secondary schools from low-income areas of Karachi.

\subsection{Objectives of the Study:}

The objective of the study is to investigate the perception and efficacy of the teachers from private secondary schools of low-income areas of Karachi to deal with the adolescent students having behaviour problems. The perception of teachers of low income areas of Karachi about the behaviour problems of the adolescent students shall be explored. Besides this, the study shall also investigate about the efficacy of these teachers to deal with the adolescent students having any sort of behaviour problems.

\subsection{Research Questions:}

The research questions guiding the study are:

RQ1: What does a teacher perceive about the behaviour disorder of adolescent students?

RQ2: What efficacy do the teachers of private secondary schools from low income areas of Karachi have to deal with such students?

\subsection{Significance of the Study:}

The findings of the study will enrich the existing literature about the topic. It shall also help the teachers in developing insight about behaviour issues of adolescent students and causes of this issue and shall help in improving their efficacy to deal with the issue. Which in turns shall be helpful in the improvement of students learning outcomes, emotional development and social skills development besides this, the study shall also help in improving the school environment, discipline and other related issues. This study shall be helpful for parents of such students to understand the causes of behaviour problems of their children. The management of 
schools shall also be benefitted by the study in order to maintain discipline and providing a congenial environment for learning. The society shall also be profited by the study in lessening the crime rate by considering the findings of the study and applying the suggestions made by the study.

\subsection{Limitation and Delimitation of the Study:}

Due to the limited availability of time, access to the schools, availability, permission and readiness of the teachers for the interview, the study was limited to the conduct of interviews of three secondary schools teachers from three private schools of lowincome areas of Karachi. Since the sample size is relatively small, the conclusive results produced by the study may not be generalize to all the private and public sector schools of Pakistan.

\subsection{Research Methodology:}

The purpose of this study is to explore the perception of teachers about the behaviour disorder of adolescent students and their efficacy to deal with these students by interviewing them in natural setting. Therefore, a qualitative research paradigm was employed, because of some of its characteristics. For instance, the first characteristic is exploring about a social phenomenon in a natural setting, which means being in a school, observing a classroom setting and interviewing people in order to get information related to the research focus. The second characteristic is that data are mostly in the form of words, pictures representing opinions and interactions.

Within the qualitative research paradigm, qualitative case study is the most suitable method for this inquiry, as literature shows that qualitative case study is the method used to study any social phenomenon focusing on a single instance that may be individuals, situation, and organization. Case study is the study of any social setting, which may be an individual, a school or a community. The study was conducted within three schools from low income areas of Karachi.

\subsection{Sampling Technique}

For the study, the most populous and largest city of Pakistan, Karachi is selected. All respondents were drawn from this city due to its socio economic disparity and nonhomogeneity. For the samples of this multiple case study, three private secondary schools of three different localities were chosen from the target population. The present study is based only on private, secondary English medium schools. This multiple case study adopted a non-random sampling technique known as purposeful purposive sampling. In this regard, the term "purposeful sampling" is used for the qualitative sampling approach where researchers intentionally select individuals and sites to learn or understand the central phenomenon. This study was conducted within the premises of selected secondary schools. The teachers 
selected for the interview were all having at least 7 to 10 years of experience of teaching and dealing adolescent students in the private schools of low-income areas of Karachi.

In this study, three private secondary schools each from different towns of Karachi were chosen. All these schools were co-educational, affiliated with the Board of Secondary Education, Karachi. The first school was Al Musleh Foundation School situated in Lyari Town, hereinafter to be refer as AMFS. Here Ms. Sehrish was interviewed. The second school was Karachi Generation School located in the North Karachi Town, hereinafter to be refer as KGS here Mr. Adnan was interviewed and the third school was Al Azhar Academy, situated in Saddar Town, hereinafter to be refer as AAA here Mr. Owais was interviewed.

\subsection{Research Instrument:}

For the purpose of study, a semi-structured interview of one secondary teacher of each selected school was conducted with the prior consent of their administration. Interviews consisted of open-ended questions.

\subsection{Instrument's Reliability and Validity:}

The instrument consisted of interview questions. In order to ensure the reliability and validity of the instrument these questions were shown to the experts in the field of social sciences and education. On the basis of their suggestions some changes were made and then the final approval was obtained from the supervisor of the research study before starting the interviews.

\subsection{Ethical Consideration:}

All the teachers for interview were selected with the prior permission of their management. And a consent letter from the IQRA University Gulshan Campus was also acquired to be presented to the management of the schools.

\subsection{Research Procedure:}

It was felt that interviews would give insight into strengthening and cross validating the findings of this study. With this intention, three semi-structured interviews were conducted with the participating teachers. Interview protocols are the most frequent qualitative research method where the researcher can explore the teaching and learning process. These are "probably the most effective ways to enlist the cooperation of the respondents" (p.372).The procedures for analyzing qualitative data that is, interviews were based on the guidelines suggested wherein the collected data was first recorded, sorted out as segments, integrated and finally 
analyzed. For this reason, firstly one pilot interview of about 35 minutes was recorded after that three main interviews for about 35-45 minutes were recorded. The device used to record the interviews was Q Mobile Model B5. Later the interviews were transcribed with the help of a transcription software, namely, Express Scribe version 5.69 for thematic analysis of each teachers' interview.

\subsection{Data Analysis and Statistical Tool:}

After coding the verbatim transcriptions, 23 excerpts of observational data, was randomly drawn and given to five raters. To get their agreement in accordance with each code given to the excerpt, so as to, measure the indices validity. Subsequently, the check- Coding reliability was computed using the online Kappa Calculator. The result of check- coding reliability is significant, as Kappa value was calculated to be 0.765. (Appendix 3)

\subsection{Findings of Qualitative data:}

This section discusses teachers' responses to various open ended questions related to their perception about the adolescent students' behaviour problems and their efficacy to deal such students. All three teachers highlighted most similar and few different behaviour problems and suggested some common strategies to deal with these problems. After segmentation, sorting, integration and analysis of the data 23 excerpts were drawn. Which are as under:

\subsubsection{Thematic Analysis: Behavioural Changes}

\subsubsection{Physical and Psychological changes}

Physical and psychological changes are prominent in most of the adolescents. One of the respondents, when asked about the major behavioural changes in the adolescent students, responded as:

"Both physical and psychological changes occur in them. They experience moodiness and become frustrated."(SSTf1)

\subsubsection{Thought process changes}

According to one respondent, the thought process of the students is changed.

They start thinking differently as compared to their previous years. He said:

"The basic changes that I have observed the students in the adolescence is basically their thought process basically get changed."(SSTm1)

\subsubsection{Want Self respect}

Another respondent was of the view that adolescent students want that their selfrespect and to be recognized. He said: 
"Actually when these students enter in adolescence age what they feel the main factor is their self-respect."(SSTm2)

\subsubsection{Girls become diffident, shy}

One respondent stated that girls in contrast to the boys become diffident and reserved. Talking about changes occurring in girls she said:

"Yeah they become reserved and at times become diffident as well. We need to make them realize that they should be confident, they start feeling shy." (SSTf1)

\subsubsection{Become confident}

One respondent pointed out that the adolescent students become more confident than their previous years, their hesitation becomes lesser and they express their views boldly.

"Their hesitation becomes lesser they become more confident while expressing their views."(SSTm1)

\subsubsection{Major Behavioural Problems}

Responding to the question regarding the major behavioural problems of the adolescent students,

\subsubsection{Break laws}

One respondent replied that these students violate the social or cultural expectations and they start breaking rules and regulations. She said:

"Their violation of social or cultural expectations. And at times when they behave like (desperados) of the class they start breaking all the laws."(SSTf1)

\subsubsection{No seriousness in studies}

Another respondent was of the view that among other major behavioral problems one of the problem is that the students are not serious in their study. He said:

"unka ziyada mind nahi rehta k hum study karen seriousness bilku lnahi hoti." (SSTm2)

\subsubsection{Start learning slang words}

The students start learning slang and abusive unacceptable language. Because of their social interaction with more people than before they adopt their way of talking and start using slang language. As one respondent stated:

"They start learning slang words." (SSTf1)

\subsubsection{Aggression}

Students become aggressive and start showing aggression over small issues. One of the respondents responded as:

"Students jo hen fight per aajate hen chhoti baat per because unke andar jo 
behaviour changes hoti hen unke andar ghussa angerness wo bohot ziyada increase ho jati hen"(SSTm2)

\subsubsection{Effects of Behavioural Problems on classroom environment}

Answering the question regarding the effects of behaviour problems of adolescent students on classroom environment,

\subsubsection{Waste of time}

The time of the classroom and fellow students is wasted due to the behaviour disorders of the adolescent students. One of the respondents said:

"This sort of behaviour mainly disturbs the teacher, distracts the teacher from the main subject. It waste the time also and you know students those who want to learn also get disturbed because of them." (SSTf1)

\subsubsection{Disturb class.}

These students disturb class and they just try to show their presence by making fun of the question asked by the teacher or by giving irrelevant answers to the question. One respondent was of the view that:

"Sometimes students make fun of the question they make their own questions and you know they just try to try to make their presence in the classroom through different attitudes" (SSTm1)

\subsubsection{Strategies to cop up with the disobeying students}

Responding to the question about the strategy to deal with the disobeying students, various strategies suggested by the respondents:

\subsubsection{Counselling}

To deal with the disobedient students, counselling is necessary to understand the reasons behind this unwanted behaviour. The answer of one respondent was:

"I try to find, what are their problems what are the reasons behind their disobeying behaviour? Why are they doing this?"(SSTf1)

\subsubsection{Give them responsibility}

Disobeying behaviour of students can change if some responsible role in the classroom is assign, as one respondent had the view:

"Make them more responsible give them some sort of responsibility in the classroom like if they are disobeying give them some responsibility" (SSTm1)

\subsubsection{Appropriate punishment}

If the disobeying student does not improve after the counselling or through other measures then sometimes, it becomes necessary to give them some appropriate punishment. One respondent was having opinion to give some appropriate punishment. 
"At times appropriate punishment becomes necessary to make them realize that what they are doing is completely unhealthy." (SSTf1)

\subsubsection{Negative reinforcement}

It may be a technique to ask disobeying student to do some task which he/she does not like to do. This technique is known as negative reinforcement. One respondent suggested using negative reinforcement to deal with such students. As he said:

"Negative reinforcement k aap wo cheez usko den k wo karna nahi chahraha acha punishment to he lekin doosri punishment he."(SSTm2)

1.14.5 How to develop positive attitude in student having problem of anxiety and depression?

To develop the positive attitude among students having problem of anxiety and depression, different strategies suggested by the respondents are:

\subsubsection{Confidence building}

This behaviour problem mainly emerges due to the shaken confidence of the child. A respondent suggested taking confidence-building measures to develop positive attitude in such students. He said

"thora sa bhi time bacon k sat spend karate hen or undo confidence date hen to aap unko bohot sari coming jo unke liye problems hen unse aap bacha sakte hen" (SSTm1)

\subsubsection{Give them importance, Involve in every task}

By giving importance and involving them in every classroom activity, we can eliminate their anxiety and depression. One of the respondents said:

"We have to make them realize that they are important for us we try to make them participate in every task."(SSTf1)

\subsubsection{Motivation}

Motivation is also a key factor to deal with the students with anxiety and depression. One of the respondent suggested that such students should be motivated to show their abilities. He said:

"Acha hamare pas ese jo bache hen wo har level pe mojood hote hen wo ese bache hote hen jin ko kehna parta he k aap parho unko aata he bol nahi paate."(SSTm2)

\subsubsection{How do teachers motivate a student with social skill deficit to mingle with peers?}

Talking about the teachers' role to deal with the students having social skill deficit problem, various suggestions were given by the respondents. Responding to this question,

\subsubsection{Give care}

Proper caring of children with social skill deficit issue can be helpful in bringing them back to the social circle. One respondent suggested providing special care to 
such students. He said:

"agar us bache ko ekmartaba ye ehsas ho jae k uski care karne wale mojood hen to wo yaqinan is situation se ya state of mind se asani se nikal sakta he." (SSTm1)

\subsubsection{Group activities}

By including such students, in-group activities where she/she is required to interact with other students can help to increase social skills in such students. One of the respondents emphasized on group activities. According to him:

"group ki planned activities bhi hosakti hen." (SSTm2)

\subsubsection{Make weaker student more responsible}

According to one respondent, making the weaker student more responsible can be a good tactic to motivate such students. She said:

"Try to make the weakest student responsible or leader."(SSTf1)

\subsubsection{Friendly behaviour}

Teacher's friendly behaviour also plays a vital role in combating the problem as if a teacher is friendly, students share their problems with the teacher which is necessary for a teacher to understand the problem and which in turns help the teacher to solve the problem. According to respondent teachers should adopt a friendly behaviour while dealing such students. She said:

"Teachers should work on them they should behave like teachers sometimes friends they should deal friendly with the students."(SSTf1)

\subsection{Discussion:}

The findings from the segmentation and thematic analysis of the qualitative data reveal that the teachers of low-income areas of the Karachi who teach and deal the adolescent students in the schools have enough understanding of the behavioural problems and causes of these problems among these students.

According to the respondents the most common changes occurring in teenage students are both physical and psychological, their thought process changes, they want their self-respect, become confident besides these girls become diffident, reserved and shy, this is in accordance with the findings reported. As far as the major behaviour problems are concerned, the respondents were of the view that commonly students become disobeying, they break laws, become aggressive, start learning slang words and they are not serious in their studies, this is found to be similar as reported in the study. Due to these behaviour problems they disturb the classroom environment by making fun of the teacher and as a consequence the precious time of the class is wasted and the fellow students have also to suffer the study loss, this is similar to the findings of the study, which found that the behavioural issues result in the wastage of time for all the students and in disturbance in the learning environment of the classroom. 
The respondents also suggested some strategies to deal with students having various behaviour problems. For disobeying students the respondents suggested different tactics, the common of these are; counselling of such students by teacher and parents, responsibility to be given to them, sometimes appropriate punishment is also necessary to make them realize that what they are doing is not healthy either for themselves or for the classroom environment, and sometimes negative reinforcement technique should be used to force such students to do what he or she doesn't like to do to prevent him/her to repeat his/her disobeying act.

For the students who are suffering from anxiety and depression, respondents suggested to take measures to boost their confidence, they may be involved in every classroom activity to show that they are important and they should be motivated to get involved in the learning activities.

The respondents suggested some strategies to motivate the students with social skill deficit to mingle with peers. Some of these are to give them proper and special care so that they realize that people are there to care for them, they may be involved in group activities so that they are supposed to interact with their peers, furthermore such students may be made more responsible or given a role of leader in order to make them work with other team members, and last but not least, the teacher should adopt a friendly behaviour with such students so that these student become closer to them and share their problems with them enabling the teacher to understand their problem and to find out the solution.

\subsection{Conclusion:}

The study has revealed that physical, emotional and psychological changes occur in the in adolescent students. Their thinking process also get changed, they want their self-respect to be honoured, they become more confident and bold, girls become shy and reserved. These changes in result produce some behaviour problems mostly in adolescent students and they are common regardless of the locality. The major behaviour problems in adolescent as reported are: Breaking of rules, regulation and discipline, lack of seriousness, use of abusive or slang language etc.

The study also found that most of the teachers who teach the adolescent students are quite aware of these issues. In addition to this, most of the teachers are capable enough to deal with these students properly. They are equipped with appropriate strategies to deal the students and help them in combating their behaviour problems. The strategies to deal with such students, suggestions given by these teachers are applicable in the secondary schools of Karachi. Furthermore, as suggested by the respondents, frequent psychological counselling sessions by psychologists should also be held for teachers to develop their positive perception of the issue and they should be provided guidance to improve their efficacy to handle the issue properly and deal such students tactfully to get them out of their 
state of mind and overcome their behaviour problems.

\subsection{Recommendations based on the Findings:}

Based on the findings and the suggestions of the respondents the causes of the behaviour problems of adolescent students should be understand clearly. To ensure the smooth functioning and congenial learning environment the teachers should adopt the strategies suggested in the discussion section. The teachers counselling sessions be held to develop teachers' perception about the issue and to make them aware about the strategies to handle these students. Parents counselling sessions may also be helpful in this regard to develop their understanding about this issue.

\subsection{Recommendations for the Future Research:}

This study is an attempt to investigate the teachers' perception about behaviour problems of adolescent students and their efficacy to deal with such students in the low-income areas of Karachi. Therefore, this study may provide an understanding into further research in this discipline for other areas of the city and in the context of Pakistan. 
Teachers' perceptions and efficacy to deal with adolescent students' behaviour disorder in schools of low-income area of Karachi

\section{References}

Anderson, E. S. Alternative school teachers' efficacy perceptions teaching students with behavioural difficulties. Dissertation, Kent State University College of Education, Health, 2014

Brookfield, S. 'Using Critical Incidents to Explore Learners' Assumptions',. in J. Mezirow (ed.) Fostering Critical Reflection in Adulthood: A Guide to Transformative and Emancipatory Learning, pp 177-193. San Francisco, CA: Jossey-Bass Publishers (1990)

Butler, M. A. "Behavioural coaching: A strategy for increasing general education teachers' skills in managing challenging behaviour." Dissertation, Urbana, Illinois, 2014.

Conchas, G., Q. \& Vigil, J., D. "Street smart school smart: Urban poverty and the education of adolescent boys." Journal of Youth and Adolescence, 43, no. 3 (2014): 502-506

Creswell, J. Educational research: Planning, conducting, and evaluating Quantitative and Qualitative research. Upper Saddle River, NJ: Merrill Prentice Hall (2002)

Fraenkel Jack, R. and Wallen Norman, E. How to design and evaluate research in education. 2nd Edition, McGraw-Hill Inc., New York (1993).

Hussein, S. A. "Behavioural problems among children attending private and community schools in Karachi." Pakistan Journal of Psychological Research 23, no. 1-2 (2008): 01-11

Infantino, J. \& Little, E. "Students' perceptions of classroom behaviour problems and the

effectiveness of different disciplinary methods." Educational Psychology 25, no. 5 (2005): 491-

508.

J.R, Christiansen. "Student / teacher relationships and school success: Perceptions of students from grades nine to twelve." A Project Submitted in Partial Fulfillment of the Requirements for the Degree., The University of Lethbridge.

Lane, N., Rowland, A., \& Beinart, H. "'No rights or wrongs, no magic solutions': Teachers' responses to bereaved adolescent students." Death Studies 38 (2014): 654-661 
Lukman, A. A., Temitayo, 0., \& Nayaya, M. A. "Management of disciplinary problems in secondary schools: Jalingo Metropolis in focus." Global Journal of Human Social Science Linguistics \& Education 13, no. 14 (2013): 07-19

Njoroge, P. M., \& Nyabuto, A. N. "Discipline as a factor in academic performance in Kenya."

Journal of Educational and Social Research 4, no. 1 (2014): 289-307

Nooruddin, S., \& Baig, S. "Student behaviour management: school leader's role in the developing country." International Journal Of Whole Schooling 11, no. 1 (2014): 19-31

Qidwai, Shah, Ishaque, \& Rahim. "Adolescent lifestyle and behaviour: A survey from a developing country." PLOS ONE . 2010. www.plosone.org.

Tsouloupas, C., N., Carson, R., L. \&MacGregor, S., K. "The development of high school teachers' efficacy in handling student misbehaviour (TEHSM)." The Journal of Educational Research 107 (2014): 230-240 